TRANSACTIONS OF THE

AMERICAN MATHEMATICAL SOCIETY

Volume 354, Number 10, Pages 4229-4243

S 0002-9947(02)03044-1

Article electronically published on May 22, 2002

\title{
HARMONIC MORPHISMS WITH ONE-DIMENSIONAL FIBRES ON EINSTEIN MANIFOLDS
}

\author{
RADU PANTILIE AND JOHN C. WOOD
}

\begin{abstract}
We prove that, from an Einstein manifold of dimension greater than or equal to five, there are just two types of harmonic morphism with onedimensional fibres. This generalizes a result of R.L. Bryant who obtained the same conclusion under the assumption that the domain has constant curvature.
\end{abstract}

\section{INTRODUCTION}

Harmonic morphisms between Riemannian manifolds are smooth maps which preserve Laplace's equation. By a basic result of B. Fuglede 9] and T. Ishihara [14], they can be characterised as harmonic maps which are horizontally weakly conformal. See 5 for a general account, and [12] for a frequently updated bibliography and other useful information on harmonic morphisms.

The first classification results for harmonic morphisms with one-dimensional fibres were obtained by P. Baird and J.C. Wood (see [5]). In [7], R.L. Bryant proved that, from a constant curvature Riemannian manifold of dimension greater than or equal to four, there exist only two types of harmonic morphisms with one-dimensional fibres (see [5] and [20] for alternative proofs). The two types can be nicely described in terms of the geometrical properties of the foliations formed by the components of the regular fibres. For this, recall [27, [19], 20] that we say a foliation produces harmonic morphisms if it can be locally defined by submersive harmonic morphisms. Then the two types of foliations which produce harmonic morphisms which appear in Bryant's theorem are the following (note that in 7] the ordering is different):

Type 1. Riemannian one-dimensional foliations locally generated by Killing vector fields 7 .

Type 2. Homothetic foliations by geodesics orthogonal to an umbilical foliation by hypersurfaces 3 .

Accordingly, a harmonic morphism with one-dimensional fibres is said to be of type 1 (respectively, type 2 ) if the components of its regular fibres form a foliation of type 1 (respectively, type 2). Note that the gradient of the dilation at regular points is horizontal for type 1 and vertical for type 2. Also, recall [2] that a harmonic morphism with one-dimensional fibres is always submersive if the domain has dimension at least five, and if the domain is of dimension four, then the set

Received by the editors December 17, 2001.

2000 Mathematics Subject Classification. Primary 58E20; Secondary 53C43.

Key words and phrases. Harmonic morphism, foliation, Einstein manifold.

The authors gratefully acknowledge that this work was done under E.P.S.R.C. grant number GR/N27897. 
of critical points is discrete (see [5] for the case when the domain has dimension three).

We prove (Theorem 2.8) that any harmonic morphism with one-dimensional fibres from an Einstein manifold of dimension greater than or equal to five is either of type 1 or of type 2, thus generalizing the result of 7]. Note that, from an Einstein four-manifold, there is just one more type of harmonic morphism with one-dimensional fibres [20, 21]; we indicate how this follows from the calculations in the present paper; see 24] for more details.

Section 1 contains some generalities on foliations which produce harmonic morphisms. The main classification result is obtained in Section 2 (Theorem 2.8). If $\left(M^{n+1}, g\right)$ is an Einstein manifold and $\varphi:\left(M^{n+1}, g\right) \rightarrow\left(N^{n}, h\right)$ is a harmonic morphism of type 2, then $\left(N^{n}, h\right)$ is also an Einstein manifold. (This follows, for example, from well-known results on warped products (see [6, Chapter 9]).) However, this is not true for a harmonic morphism of type 1. In Section 3 we refine the classification of harmonic morphisms with one-dimensional fibres in the case when both the domain and codomain are Einstein manifolds. The result (Theorem 3.1) shows that only two well-known constructions (see [6, Chapter 9]), illustrated by the Hopf fibrations and suitable warped products over Einstein manifolds, can occur. In the Appendix we prove a fact on horizontally holomorphic submersions which is needed in the proof of Theorem 3.1.

\section{Foliations Which PRODUCE HARMONIC MORPHisms}

Let $\mathcal{V}$ be (the tangent bundle of) a smooth foliation on a smooth Riemannian manifold $(M, g)$ and set $\mathcal{H}=\mathcal{V}^{\perp}$. Recall (see [19], 20] ) that we say that $\mathcal{V}$ produces harmonic morphisms if it can be locally defined by submersive harmonic morphisms. A foliation of codimension two produces harmonic morphisms if and only if it is a conformal foliation with minimal leaves [27]. For foliations of codimension not equal to two which produce harmonic morphisms, we have the following characterisation of R.L. Bryant [7] (see also [5], 19] or [20] ).

Proposition 1.1. Let $\mathcal{V}$ be a foliation of $\operatorname{codim} \mathcal{V}=n \neq 2$ on the Riemannian manifold $(M, g)$. Then the following assertions are equivalent:

(i) $\mathcal{V}$ produces harmonic morphisms;

(ii) $\mathcal{V}$ is a conformal foliation and the one-form

$$
\beta=(n-2) \operatorname{trace}\left(B^{\mathcal{H}}\right)^{b}-n \operatorname{trace}\left(B^{\mathcal{V}}\right)^{b}
$$

is closed. (Here $\mathcal{H}=\mathcal{V}^{\perp}$, and $B^{\mathcal{H}}$ (respectively, $B^{\mathcal{V}}$ ) denotes the second fundamental form of $\mathcal{H}$ (respectively, $\mathcal{V})$; note that we shall denote by the same letter $\mathcal{H}$ (respectively, $\mathcal{V}$ ) the horizontal (respectively, vertical) distribution and the orthogonal projection onto it).

We shall also need the following definition ([19], 20]).

Definition 1.2. We say that a foliation is homothetic if it can be locally defined by horizontally homothetic submersions (i.e., horizontally conformal submersions whose dilations are constant along horizontally curves).

See [5], [19] and [20] for more information on homothetic foliations.

The following result answers a question of P. Baird. (See [5] for a proof.)

Proposition 1.3. Let $(M, g)$ be a real-analytic Riemannian manifold, and let $\mathcal{V}$ be a foliation which produces harmonic morphisms on $(M, g)$. 
Then $\mathcal{V}$ is a real-analytic foliation. Moreover, if $\operatorname{codim} \mathcal{V} \neq 2$, then any harmonic morphism produced by $\mathcal{V}$ is a real-analytic map onto a real-analytic Riemannian manifold. If $\operatorname{codim} \mathcal{V}=2$, then $\mathcal{V}$ is locally defined by real-analytic submersive harmonic morphisms onto real-analytic Riemannian two-manifolds.

Corollary 1.4. Let $\varphi:(M, g) \rightarrow(N, h)$ be a submersive harmonic morphism from a real-analytic manifold onto a smooth manifold.

If $\operatorname{dim} N \neq 2$, then there exists a real-analytic structure on $N$ with respect to which $\varphi$ and $h$ are real-analytic. If $\operatorname{dim} N=2$, then $\varphi$ is real-analytic with respect to the real-analytic structure on $N$ induced by the conformal structure of $h$.

Remark 1.5. In Corollary 1.4, if $\operatorname{dim} N=2$, then we can remove the hypothesis that $\varphi$ is submersive; note, however, that the metric of $N$ may always be chosen such that it is not real-analytic (just apply a suitable conformal deformation).

If $\operatorname{dim} N \geq 3$, we do not know if the hypothesis that $\varphi$ is submersive can be removed.

\section{THE PROOF OF THE MAIN THEOREM}

We shall need two straightforward lemmas. For the reader's convenience we include their proofs here.

Lemma 2.1. For $n \geq 1$, let $P: \mathbb{C}^{n} \times \mathbb{C} \rightarrow \mathbb{C}$ be defined by

$$
P(\mathbf{a}, \lambda)=\lambda^{n}+a_{1} \lambda^{n-1}+\cdots+a_{n}, \quad\left(\mathbf{a}=\left(a_{1}, \ldots, a_{n}\right) \in \mathbb{C}^{n}, \lambda \in \mathbb{C}\right) .
$$

Let $\left(\mathbf{a}^{(k)}\right) \subseteq \mathbb{C}^{n}$ be a convergent sequence.

Then the set $S=\left\{\lambda \in \mathbb{C} \mid \exists k: P\left(\mathbf{a}^{(k)}, \lambda\right)=0\right\}$ is bounded.

Proof. Let $\widetilde{P}: \mathbb{C}^{n} \times \mathbb{C} P^{1} \rightarrow \mathbb{C} P^{1}$ be defined by

$$
\widetilde{P}\left(\mathbf{a},\left[\lambda_{0}, \lambda_{1}\right]\right)=\left[\lambda_{0}^{n}, \lambda_{1}^{n}+a_{1} \lambda_{0} \lambda_{1}^{n-1}+\cdots+a_{n} \lambda_{0}^{n}\right], \quad\left(\mathbf{a} \in \mathbb{C}^{n},\left[\lambda_{0}, \lambda_{1}\right] \in \mathbb{C} P^{1}\right) .
$$

Obviously, $S=\bigcup_{k} S_{k}$ where $S_{k}=\left\{\lambda \in \mathbb{C} \mid \widetilde{P}\left(\mathbf{a}^{(k)},[1, \lambda]\right)=[1,0]\right\}$; suppose that $S$ is unbounded. For each $k$, let $\lambda^{(k)} \in S_{k}$ be such that $|\lambda| \leq\left|\lambda^{(k)}\right|$ for every $\lambda \in S_{k}$. Because $S$ is unbounded we must have that $\left(\lambda^{(k)}\right)$ is unbounded. By passing to a subsequence, if necessary, we can suppose that $\lambda^{(k)} \rightarrow \infty$.

Because $\lambda^{(k)} \in S_{k}$ we have $\widetilde{P}\left(\mathbf{a}^{(k)},\left[1, \lambda^{(k)}\right]\right)=[1,0]$. But

$$
\lim _{k \rightarrow \infty} \widetilde{P}\left(\mathbf{a}^{(k)},\left[1, \lambda^{(k)}\right]\right)=\widetilde{P}(\mathbf{b},[0,1])=[0,1]
$$

where $\mathbf{b}=\lim _{k \rightarrow \infty} \mathbf{a}^{(k)}$, a contradiction. Hence $S$ must be bounded.

For the second lemma we need the following definition.

Definition 2.2. Let $E \rightarrow M$ be a vector bundle endowed with a Riemannian metric $h$, and let $T \in \Gamma\left(\odot^{2} E^{*}\right)$. Say that $T$ can be consistently diagonalized at $x_{0} \in M$ if there exists an open neighbourhood $U$ of $x_{0}$ and a local orthonormal frame $\left\{e^{j}\right\}$ on $U$ for $E^{*}$ such that

$$
T=\sum_{j, k} \mu_{j} \delta_{j k} e^{j} \otimes e^{k}
$$

for some smooth functions $\mu_{j}: U \rightarrow \mathbb{R}$.

A similar definition can be made for a field of selfadjoint endomorphisms $\widetilde{T} \in$ $\Gamma($ End $E)$. 
Lemma 2.3. Let $E \rightarrow M$ be a vector bundle endowed with a Riemannian metric $h$, and let $T \in \Gamma\left(\odot^{2} E^{*}\right)$. Then $T$ can be consistently diagonalized at each point of a dense open subset of $M$.

Proof. The proof is by induction on the rank (fibre dimension) of $E$.

For $\operatorname{rank} E=1$ the lemma is trivial.

Suppose that the assertion of the lemma is true for $\operatorname{rank} E<n$; we shall prove that the assertion is true for $\operatorname{rank} E=n$.

Let $P(x, \lambda)=P_{x}(\lambda)$ be the characteristic polynomial of $T_{x}$, with respect to $h_{x}$ $(x \in M)$. For $p=1, \ldots, n$, set

$$
G_{p}=\left\{x \in M \mid P_{x} \text { has a root of order at most } p\right\},
$$

and set $G_{0}=\emptyset$. Because

$$
M=\bigcup_{p=1}^{n} \bar{G}_{p} \backslash \bar{G}_{p-1} \subseteq \bigcup_{p=1}^{n} \overline{G_{p} \backslash \bar{G}_{p-1}}=\overline{\bigcup_{p=1}^{n} G_{p} \backslash \bar{G}_{p-1}} \subseteq M,
$$

we have that

$$
M=\overline{\bigcup_{p=1}^{n} G_{p} \backslash \bar{G}_{p-1}}
$$

where $\bar{A}$ denotes the closure of the set $A$. To complete the proof, it suffices to prove that each $x \in \bigcup_{p=1}^{n} G_{p} \backslash \bar{G}_{p-1}$ has an open neighbourhood $U$ such that $\left.E\right|_{U}=E_{1} \oplus E_{2}$ where $E_{1}$ and $E_{2}$ are complementary orthogonal vector subbundles of $E$ of positive rank such that $\left.T\right|_{E_{1} \otimes E_{2}}=0$.

Let $p \in\{1, \ldots, n\}$ be such that $G_{p} \backslash \bar{G}_{p-1} \neq \emptyset$ and let $x_{0} \in G_{p} \backslash \bar{G}_{p-1}$. Let $\lambda_{0}$ be a root of $P_{x_{0}}$ of order at most $p$. Because $x_{0}$ is not in $\bar{G}_{p-1}$ we have that $\lambda_{0}$ has order $p$. Then, by the Malgrange Preparation Theorem (see [10, Chapter IV] ), in an open neighbourhood $U \subseteq M \backslash \bar{G}_{p-1}$ of $x_{0}$, we have $P(x, \lambda)=Q(x, \lambda) R(x, \lambda)$ where $Q$ is a polynomial of degree $p$ in $\lambda$ such that $Q\left(x_{0}, \lambda\right)=\left(\lambda-\lambda_{0}\right)^{p}$ and $R\left(x_{0}, \lambda_{0}\right) \neq 0$. From the fact that $P$ and $Q$ are both polynomials in $\lambda$ (with coefficients smooth functions of $x$ ), it follows that $R$ is also polynomial in $\lambda$.

We shall show that there exists an open neighbourhood $V \subseteq U$ of $x_{0}$ such that, for each $x \in V, Q_{x}$ has a root of order $p$. Suppose not. Let $\left(x^{(k)}\right) \subseteq U$ be such that $\lim _{k \rightarrow \infty} x^{(k)}=x_{0}$ and, for each $k$, there exists $\mu^{(k)} \in \mathbb{R}$ such that $Q\left(x^{(k)}, \mu^{(k)}\right)=0$ with $\mu^{(k)}$ a root of $Q_{x^{(k)}}$ of order less than $p$. Obviously, $\mu^{(k)}$ is also a root of $P_{x^{(k)}}$ and, because $x^{(k)} \in U \subseteq M \backslash \bar{G}_{p-1}, \mu^{(k)}$ is a root of order at least $p$ of $P_{x^{(k)}}$. Hence $R\left(x^{(k)}, \mu^{(k)}\right)=0$. Now, by Lemma 2.1, the sequence $\left(\mu^{(k)}\right)$ is bounded and hence, by passing to a subsequence if necessary, we can suppose that $\lim _{k \rightarrow \infty} \mu^{(k)}=\mu_{0}$ with $\mu_{0} \in \mathbb{R}$. Then $R\left(x_{0}, \mu_{0}\right)=\lim _{k \rightarrow \infty} R\left(x^{(k)}, \mu^{(k)}\right)=0$ and also $Q\left(x_{0}, \mu_{0}\right)=\lim _{k \rightarrow \infty} Q\left(x^{(k)}, \mu^{(k)}\right)=0$. Because $R\left(x_{0}, \lambda_{0}\right) \neq 0$ we have that $\mu_{0} \neq \lambda_{0}$. But this implies that $\lambda_{0}$ is not a root of order $p$ of $Q_{x_{0}}$. It follows that, in an open neighbourhood $V$ of $x_{0}$, we have that $Q_{x}$ has only roots of order $p$ for any $x \in V$. Thus we can write $Q(x, \lambda)=(\lambda-\mu(x))^{p},((x, \lambda) \in V \times \mathbb{R})$, where $\mu(x)$ is the root of $\partial^{p-1} Q / \partial \lambda^{p-1}(x, \cdot)$, so that $\mu$ is smooth. Hence

$$
P(x, \lambda)=(\lambda-\mu(x))^{p} R(x, \lambda), \quad((x, \lambda) \in V \times \mathbb{R}) .
$$


Moreover, because $\partial^{p} P / \partial \lambda^{p}\left(x_{0}, \lambda_{0}\right) \neq 0$, we can suppose that $\partial^{p} P / \partial \lambda^{p}(x, \mu(x))$ is nonzero for any $x \in V$. It follows that $\mu(x)$ is an eigenvalue of order $p$ for $T_{x}$ for any $x \in V$.

Let $\left(E_{1}\right)_{x}$ be the eigenspace of $\mu(x)$ and let $\left(E_{2}\right)_{x}$ be its orthogonal complement. It is easy to see that $E_{j}=\bigcup_{x \in V}\left(E_{j}\right)_{x},(j=1,2)$, are smooth subbundles of $E$ which have the required properties. The lemma follows.

Let $\varphi:\left(M^{n+1}, g\right) \rightarrow\left(N^{n}, h\right)$ be a submersive harmonic morphism between Riemannian manifolds of dimension $n+1$ and $n$, respectively. Let $\mathcal{V}$ be the foliation formed by the components of the fibres of $\varphi$, and let $V \in \Gamma(\mathcal{V})$ be a local vertical vector field such that $g(V, V)=\lambda^{2 n-4}$. Then let $\theta$ be the vertical dual of $V$ (i.e., $\theta(V)=1$ and $\operatorname{ker} \theta=\mathcal{H}$ with $\left.\mathcal{H}=\mathcal{V}^{\perp}\right)$. Note that, at each point, both $V$ and $\theta$ are uniquely determined up to sign. Therefore, we can globally define a Riemannian metric $\bar{h}$ on $M^{n+1}$ by

$$
\bar{h}=\varphi^{*}(h)+\theta^{2} .
$$

Note that $\varphi:(M, \bar{h}) \rightarrow(N, h)$ is a Riemannian submersion with geodesic fibres. It follows that $V$ is an infinitesimal automorphism of $\mathcal{H}$; equivalently,

$$
[V, X]=0 \quad \text { for any basic vector field } X \in \Gamma(\mathcal{H}) \text {. }
$$

Set $\Omega=\mathrm{d} \theta$; from (2.1) it follows easily that $\Omega$ is basic, equivalently, $i_{V} \Omega=0$ and $\mathcal{L}_{V} \Omega=0$. Also, $\mathcal{H}$ is integrable if and only if $\Omega=0$.

To simplify the notation, from now on we shall denote by the same letter $h$ the induced metric $\bar{h}$ on $M$. Obviously, $\left.h\right|_{\mathcal{H}}$ is basic.

From [20] Lemma B.1.5] (see [19] Lemma 5.2, (4.4)] or [5] ) we recall the following:

Lemma 2.4. Let $n \geq 1$ and let $\varphi:\left(M^{n+1}, g\right) \rightarrow\left(N^{n}, h\right)$ be a submersive harmonic morphism between Riemannian manifolds of dimension $n+1$ and $n$, respectively. Let $\lambda=e^{\sigma}$ be the dilation of $\varphi$.

Let ${ }^{M}$ Ricci denote the Ricci tensor of $(M, g)$ and ${ }^{N}$ Ricci the Ricci tensor of $(N, h)$; then,

$$
\begin{gathered}
{ }^{M} \operatorname{Ricci}(X, Y)=\left({ }^{N} \operatorname{Ricci}\right)\left(\varphi_{*} X, \varphi_{*} Y\right)-\frac{1}{2} e^{(2 n-2) \sigma} h\left(i_{X} \Omega, i_{Y} \Omega\right) \\
-e^{-2 \sigma}\left(\Delta^{M} \sigma\right) h(X, Y)-(n-1)(n-2) X(\sigma) Y(\sigma), \\
{ }^{M_{R} \operatorname{Ricci}(X, V)=} \frac{1}{2} e^{(2 n-2) \sigma}\left({ }^{h} \mathrm{~d}^{*} \Omega\right)(X)+(n-1) e^{(2 n-2) \sigma} \Omega\left(X, \operatorname{grad}_{h} \sigma\right) \\
+(n-1) X(V(\sigma))-(n-1)(n-2) X(\sigma) V(\sigma), \\
{ }^{M} \operatorname{Ricci}(V, V)=(n-2) e^{(2 n-4) \sigma} \Delta^{M} \sigma+2(n-1) V(V(\sigma)) \\
-(3 n-4)(n-1) V(\sigma)^{2}+\frac{1}{4} e^{(4 n-4) \sigma}|\Omega|_{h}^{2} .
\end{gathered}
$$

where ${ }^{h} \mathrm{~d}^{*}$ denotes the codifferential on $(M, h)$.

We shall also need the following result of [19, Theorem 5.7, Corollary 5.9] (see also [20]).

Proposition 2.5. Let $(M, g)$ be an Einstein manifold of dimension at least 4, and let $\mathcal{V}$ be a one-dimensional foliation which produces harmonic morphisms on $(M, g)$. Suppose that, either, the orthogonal complement $\mathcal{H}$ of $\mathcal{V}$ is integrable, or, $\mathcal{V}$ is a homothetic foliation.

Then either, 
(i) $\mathcal{V}$ is a Riemannian foliation locally generated by Killing vector fields, or

(ii) $\mathcal{V}$ is a homothetic foliation by geodesics orthogonal to an umbilical foliation by hypersurfaces.

Finally, we shall also need the following result from [21 Proposition 1.12] (see also [20]).

Proposition 2.6. Let $(M, g)$ be an Einstein manifold, and let $\mathcal{V}$ be a one-dimensional foliation of codimension not equal to two which produces harmonic morphisms on $(M, g)$.

Then the following assertions are equivalent:

(i) $\mathcal{V}$ has basic mean curvature form;

(ii) $\mathcal{V}$ is a homothetic foliation.

Remark 2.7. Let $\varphi:(M, g) \rightarrow(N, h)$ be a submersive harmonic morphism. Let $\mathcal{V}=\operatorname{ker} \varphi_{*}$ and let $\lambda$ be the dilation of $\varphi$. Then, from the fundamental formula of P. Baird and J. Eells (see, for example, [5] or [19]), it follows easily that $\operatorname{trace}\left(B^{\mathcal{V}}\right)^{b}$ is basic if and only if $X(\log \lambda)$ is a basic function for any basic vector field $X \in \Gamma(\mathcal{H})$.

We now state the main theorem.

Theorem 2.8. Let $(M, g)$ be an Einstein manifold of dimension at least 5, and let $\mathcal{V}$ be a one-dimensional foliation which produces harmonic morphisms on $(M, g)$. Then either,

(i) $\mathcal{V}$ is a Riemannian foliation locally generated by Killing vector fields, or

(ii) $\mathcal{V}$ is a homothetic foliation by geodesics orthogonal to an umbilical foliation by hypersurfaces.

Since by [2] any harmonic morphism with one-dimensional fibres from a Riemannian manifold of dimension at least 5 is submersive, from Theorem 2.8 we obtain the following.

Corollary 2.9. Let $\left(M^{n+1}, g\right)$ be an Einstein manifold of dimension $n+1 \geq 5$, and let $\varphi:\left(M^{n+1}, g\right) \rightarrow\left(N^{n}, h\right)$ be a nonconstant harmonic morphism to a Riemannian manifold of dimension $n$.

Then either,

(i) the components of the fibres of $\varphi$ form a Riemannian foliation locally tangent to nowhere zero Killing vector fields, or

(ii) $\varphi$ is a horizontally homothetic submersion with geodesic fibres orthogonal to an umbilical foliation by hypersurfaces.

Remark 2.10. 1) Note that, in case (i), $\varphi$ is not, in general, a Riemannian submersion. In fact, its dilation is proportional to $|V|^{1 /(n-2)}$ where $V$ is a Killing vector field tangent to the fibres 7] (see also [5], [19], [20]).

2) Since, in case (ii), the hypersurfaces are the level hypersurfaces of $\lambda$ which is an isoparametric function, the fibres of $\varphi$ are orthogonal to an isoparametric function [1] (see [5]).

Proof of Theorem [2.8. By Proposition [1.3, if the horizontal distribution $\mathcal{H}$ is integrable on an open subset of $M$, then $\mathcal{H}$ is integrable on $M$. Thus, by Proposition 2.5, it is sufficient to prove the case when $\mathcal{H}$ is nowhere integrable. Also, writing $n+1=\operatorname{dim} M$, we can suppose that the leaves of $\mathcal{V}$ are the fibres of a harmonic morphism $\varphi:\left(M^{n+1}, g\right) \rightarrow\left(N^{n}, \bar{h}\right)$, where $\operatorname{dim} N=n$ with $n \geq 4$, and from now on we shall use the notations of Lemma 2.4. Let $\Omega^{2} \in \Gamma(\operatorname{End}(\mathcal{H}))$ be 
the field of selfadjoint negative semi-definite endomorphisms of $\left(\mathcal{H},\left.h\right|_{\mathcal{H}}\right)$ defined by $h\left(\Omega^{2}(X), Y\right)=-h\left(i_{X} \Omega, i_{Y} \Omega\right)$ for horizontal $X$ and $Y$.

By Lemma [2.3, $\Omega^{2}$ can be consistently diagonalized on a dense open subset of $M$; let $x_{0} \in M$ be a point of this subset. There is an open neighbourhood $U$ of $x_{0}$ and an orthonormal frame $\left\{X_{1}, \ldots, X_{n}\right\}$ for $\left(\mathcal{H},\left.h\right|_{\mathcal{H}}\right)$ over $U$ such that $\Omega^{2}\left(X_{i}\right)=-\mu_{i}^{2} X_{i}$ for some continuous functions $\mu_{i}: U \rightarrow[0, \infty)$ with $\mu_{i}^{2}$ smooth. Because $\Omega$ and $\left.h\right|_{\mathcal{H}}$ are basic, we also have that $\Omega^{2}$ is basic; hence the $\mu_{i}$ are basic as well. We can thus suppose that the $X_{i}$ are basic.

From (2.2) we have

$$
{ }^{N} \operatorname{Ricci}\left(\varphi_{*} X_{i}, \varphi_{*} X_{j}\right)=(n-1)(n-2) X_{i}(\sigma) X_{j}(\sigma) \quad(i, j=1, \ldots, n, i \neq j) .
$$

We have the following alternative. Either

(1) there exists $x \in U$ and distinct $j_{1}, j_{2}, j_{3}$ such that $X_{j_{k}}(\sigma)_{x} \neq 0(k=1,2,3)$, or

(2) for any $x \in U$ there are at most two distinct values of $j$, say $j_{1}, j_{2}$ such that $X_{j_{k}}(\sigma)_{x} \neq 0 \quad(k=1,2)$.

Suppose that (1) holds. By (2.5) we have that $X_{i}(\sigma) X_{j}(\sigma)$ is basic for any $i \neq j$. Hence $X_{j_{1}}(\sigma)^{2} X_{j_{2}}(\sigma)^{2} X_{j_{3}}(\sigma)^{2}$ is basic, and, because $X_{j_{k}}(\sigma) \neq 0$ on some open subset of $U$, we have that $X_{j_{k}}(\sigma)$ is basic $(k=1,2,3)$. Thus, if (1) holds, $X_{i}(\sigma)$ is basic for all $i=1, \ldots, n$, on some open subset of $U$. Then, by Proposition 1.3 and Proposition 2.6 (see also Remark 2.7), $\mathcal{V}$ is homothetic on $M$ and the proof follows from Proposition 2.5.

Suppose that (2) holds. If $X_{j}(\sigma)=0$ for all $j=1, \ldots, n$, then $\mathcal{V}$ is a homothetic foliation and the proof of the theorem follows from Proposition [2.5. Therefore we can suppose that, after renumbering if necessary, we have $X_{1}(\sigma)_{x} \neq 0$ at some point $x \in U$. Then this holds on some open subset of $U$. Then, either $X_{j}(\sigma)=0$ for $j=2, \ldots, n$ on some open subset of $U$, or there exists a point $x \in U$ such that, after renumbering if necessary, $X_{1}(\sigma)_{x} \neq 0$ and $X_{2}(\sigma)_{x} \neq 0$. In the latter case, because (2) holds, we must have that $X_{j}(\sigma)=0(j=3, \ldots, n)$ on some open subset of $U$. It follows that there exists an open subset $U_{1}$ of $U$ such that $X_{j}(\sigma)=0(j \geq 3)$. From now on we shall work on $U_{1}$.

By (2.2) we have

$$
\begin{aligned}
c^{M} e^{-2 \sigma}={ }^{N} \operatorname{Ricci}\left(\varphi_{*} X_{i}, \varphi_{*} X_{i}\right)-\frac{1}{2} e^{(2 n-2) \sigma} \mu_{i}^{2} & \\
- & -e^{-2 \sigma} \Delta^{M} \sigma-(n-1)(n-2) X_{i}(\sigma)^{2} \quad(i=1, \ldots, n) .
\end{aligned}
$$

From (2.6) we get

$$
\begin{aligned}
{ }^{N} \operatorname{Ricci}\left(\varphi_{*} X_{i}, \varphi_{*} X_{i}\right)-{ }^{N} \operatorname{Ricci}\left(\varphi_{*} X_{j}, \varphi_{*} X_{j}\right)-\frac{1}{2} e^{(2 n-2) \sigma}\left(\mu_{i}^{2}-\mu_{j}^{2}\right) \\
-(n-1)(n-2)\left(X_{i}(\sigma)^{2}-X_{j}(\sigma)^{2}\right)=0 \quad(i, j=1, \ldots, n) .
\end{aligned}
$$

From (2.7) it follows that $\frac{1}{2} e^{(2 n-2) \sigma}\left(\mu_{i}^{2}-\mu_{j}^{2}\right)$ is basic for $i, j \geq 3$. Thus, if $\mu_{i} \neq \mu_{j}$ at some point for some $i, j \geq 3, i \neq j$, then $e^{\sigma}$ is basic and so $\mathcal{V}$ is Riemannian on some open subset of $M$; hence, by Proposition 1.3, $\mathcal{V}$ is Riemannian on $(M, g)$. It remains to consider the case when $\mu_{3}=\ldots=\mu_{n}=\mu$ for some function $\mu$.

Now, either, $\mu_{1}=\mu_{2}$ on some open subset, or, $\mu_{1} \neq \mu_{2}$ on a dense open subset. In the former case, by (2.7), we have that $X_{1}(\sigma)^{2}-X_{2}(\sigma)^{2}$ is basic on some open subset. But, by (2.5),$X_{1}(\sigma) X_{2}(\sigma)$ is also basic, and hence $X_{1}(\sigma), X_{2}(\sigma)$ are basic on some open subset. Since $X_{j}(\sigma)=0$ for $j \geq 3, \mathcal{V}$ has basic mean curvature form. Then, by Proposition [2.6], $\mathcal{V}$ is homothetic on some open subset and hence, 
by Proposition [1.3, $\mathcal{V}$ is homothetic on $(M, g)$; the proof of the theorem follows from Proposition [2.5.

It remains to consider the case when $\mu_{1} \neq \mu_{2}$. Because $\Omega$ is skew-symmetric, at each point $x$, for any $i \in\{1, \ldots, n\}$ with $\mu_{i}(x) \neq 0$ there exists $j \in\{1, \ldots, n\}$, $j \neq i$, such that $\mu_{i}(x)=\mu_{j}(x)$. Hence, at each point $x$, we have that either $\mu_{1}(x)=\mu(x)$ and $\mu_{2}(x) \neq \mu(x)$ or $\mu_{1}(x) \neq \mu(x)$ and $\mu_{2}(x)=\mu(x)$. Suppose that $\mu_{1}(x) \neq \mu(x)$; then this holds at all points of an open subset, and on that subset we must have $\mu_{2}=\mu$. Moreoever, because $\Omega$ is skew-symmetric, we must have $\mu_{1}=0$ and so $\mu$ is not identically zero; in particular, $n-1$ is even, i.e., $n=2 k+1$ for some integer $k \geq 1$.

From (2.7) we get

$$
{ }^{N} \operatorname{Ricci}\left(\varphi_{*} X_{2}, \varphi_{*} X_{2}\right)-{ }^{N} \operatorname{Ricci}\left(\varphi_{*} X_{3}, \varphi_{*} X_{3}\right)=(n-1)(n-2) X_{2}(\sigma)^{2} ;
$$

hence, $X_{2}(\sigma)$ is basic. Thus, if $X_{2}(\sigma) \neq 0$, since $X_{1}(\sigma) X_{2}(\sigma)$ is basic, we deduce that $X_{1}(\sigma)$ is also basic and the proof follows as before. There remains the case when $X_{2}(\sigma)=0$ which we now consider. Summing up the previous discussion, we have that $n=2 k+1, k \geq 1$, and we are now on an open subset on which we have the following:

$$
\begin{aligned}
& \mu_{1}=0, \mu_{2}=\ldots=\mu_{n}=\mu, \\
& X_{2}(\sigma)=\ldots=X_{n}(\sigma)=0, \\
& \mu \text { and } X_{1}(\sigma) \text { are not identically zero. }
\end{aligned}
$$

Moreover, we can assume that $\mu$ and $X_{1}(\sigma)$ are nowhere zero. Furthermore, because $\Omega^{2}\left(X_{1}\right)=-\mu_{1}^{2}$, we have that $\left|i_{X_{1}} \Omega\right|_{h}^{2}=\mu_{1}^{2}=0$. Hence $i_{X_{1}} \Omega=0$, equivalently $i_{\operatorname{grad}_{h} \sigma} \Omega=0$.

From this and (2.3) it follows that we have for $i=1, \ldots, n$,

$$
0=\frac{1}{2} e^{(2 n-2) \sigma}\left({ }^{h} \mathrm{~d}^{*} \Omega\right)\left(X_{i}\right)+(n-1) X_{i}(V(\sigma))-(n-1)(n-2) X_{i}(\sigma) V(\sigma) \text {. }
$$

Next, we compute $\left({ }^{h} \mathrm{~d}^{*} \Omega\right)\left(X_{1}\right)$ :

$$
\begin{aligned}
\left({ }^{h} \mathrm{~d}^{*} \Omega\right)\left(X_{1}\right) & =-\sum_{j=1}^{2 k+1}\left(\nabla_{X_{j}} \Omega\right)\left(X_{j}, X_{1}\right) \\
& =-\sum_{j=1}^{2 k+1}\left\{X_{j}\left(\Omega\left(X_{j}, X_{1}\right)\right)-\Omega\left(\nabla_{X_{j}} X_{j}, X_{1}\right)-\Omega\left(X_{j}, \nabla_{X_{j}} X_{1}\right)\right\} \\
& =\sum_{j=1}^{2 k+1} \Omega\left(X_{j}, \nabla_{X_{j}} X_{1}\right)=\sum_{j=2}^{2 k+1} \Omega\left(X_{j}, \nabla_{X_{j}} X_{1}\right) \\
& =\sum_{j=1}^{k}\left\{\Omega\left(X_{2 j}, \nabla_{X_{2 j}} X_{1}\right)+\Omega\left(X_{2 j+1}, \nabla_{X_{2 j+1}}, X_{1}\right)\right\} .
\end{aligned}
$$

We can choose a basic orthonormal local frame $\left\{X_{1}, X_{2}, \ldots, X_{2 k+1}\right\}$ such that

$$
\left(\Omega_{i j}\right)=\left(\begin{array}{cccccc}
0 & 0 & 0 & \ldots & 0 & 0 \\
0 & 0 & -\mu & \ldots & 0 & 0 \\
0 & \mu & 0 & \ldots & 0 & 0 \\
\ldots & \ldots & \ldots & \ldots & \ldots & \ldots \\
0 & 0 & 0 & \ldots & 0 & -\mu \\
0 & 0 & 0 & \ldots & \mu & 0
\end{array}\right) .
$$


Then, from the above calculation we have

$$
\begin{aligned}
\left({ }^{h} \mathrm{~d}^{*} \Omega\right)\left(X_{1}\right) & =\sum_{j=1}^{k}\left\{h\left(\nabla_{X_{2 j}} X_{1}, X_{2 j+1}\right) \Omega_{2 j, 2 j+1}+h\left(\nabla_{X_{2 j+1}} X_{1}, X_{2 j}\right) \Omega_{2 j+1,2 j}\right\} \\
& =\sum_{j=1}^{k}\left\{-\mu h\left(\nabla_{X_{2 j}} X_{1}, X_{2 j+1}\right)+\mu h\left(\nabla_{X_{2 j+1}} X_{1}, X_{2 j}\right)\right\} \\
& =\mu \sum_{j=1}^{k}\left\{h\left(X_{1}, \nabla_{X_{2 j}} X_{2 j+1}\right)-h\left(X_{1}, \nabla_{X_{2 j+1}} X_{2 j}\right)\right\} \\
& =\mu \sum_{j=1}^{k} h\left(X_{1},\left[X_{2 j}, X_{2 j+1}\right]\right) .
\end{aligned}
$$

Recall that $X_{j}(\sigma)=0$ for all $j \geq 2$; hence,

$$
\begin{aligned}
{\left[X_{2 j}, X_{2 j+1}\right](\sigma)=0 } & \Longleftrightarrow-\mathcal{V}\left[X_{2 j}, X_{2 j+1}\right](\sigma)=\mathcal{H}\left[X_{2 j}, X_{2 j+1}\right](\sigma) \\
& \Longleftrightarrow \Omega\left(X_{2 j}, X_{2 j+1}\right) V(\sigma)=h\left(\left[X_{2 j}, X_{2 j+1}\right], \mathcal{H}\left(\operatorname{grad}_{h} \sigma\right)\right) \\
& \Longleftrightarrow-\mu V(\sigma)=h\left(\left[X_{2 j}, X_{2 j+1}\right], X_{1}\right) X_{1}(\sigma) \\
& \Longleftrightarrow h\left(\left[X_{2 j}, X_{2 j+1}\right], X_{1}\right)=-\mu \frac{V(\sigma)}{X_{1}(\sigma)} .
\end{aligned}
$$

It follows from the last equation that

$$
\left({ }^{h} \mathrm{~d}^{*} \Omega\right)\left(X_{1}\right)=-k \mu^{2} \frac{V(\sigma)}{X_{1}(\sigma)} .
$$

From (2.8) and (2.10) we get

$$
0=-\frac{1}{2} k \mu^{2} e^{4 k \sigma} \frac{V(\sigma)}{X_{1}(\sigma)}+2 k X_{1}(V(\sigma))-2 k(2 k-1) X_{1}(\sigma) V(\sigma),
$$

which is equivalent to

$$
\mu^{2} e^{4 k \sigma} V(\sigma)=4 X_{1}(\sigma) X_{1}(V(\sigma))-4(2 k-1) X_{1}(\sigma)^{2} V(\sigma) .
$$

From (2.7) with $i=1, j=2$, we get that

$$
\frac{1}{2} e^{4 k \sigma} \mu^{2}-2 k(2 k-1) X_{1}(\sigma)^{2} \quad \text { is basic, }
$$

and hence on differentiating this with respect to $V$ and using (2.1), we obtain

$$
2 k e^{4 k \sigma} V(\sigma) \mu^{2}-4 k(2 k-1) X_{1}(\sigma) X_{1}(V(\sigma))=0,
$$

which is equivalent to

$$
\mu^{2} e^{4 k \sigma} V(\sigma)=2(2 k-1) X_{1}(\sigma) X_{1}(V(\sigma)) .
$$

From (2.11) and (2.12) we get that

$$
4 X_{1}(\sigma) X_{1}(V(\sigma))-4(2 k-1) X_{1}(\sigma)^{2} V(\sigma)=2(2 k-1) X_{1}(\sigma) X_{1}(V(\sigma)),
$$

which, because $X_{1}(\sigma)$ is nowhere zero, is equivalent to

$$
X_{1}(V(\sigma))=-\frac{2(2 k-1)}{2 k-3} X_{1}(\sigma) V(\sigma) .
$$


From (2.12) and (2.13) it follows that

$$
\mu^{2} e^{4 k \sigma} V(\sigma)=-\frac{4(2 k-1)^{2}}{2 k-3} X_{1}(\sigma)^{2} V(\sigma),
$$

which, if $\mathcal{V}$ is not Riemannian (equivalently, $V(\sigma) \neq 0$ ), implies that

$$
\mu^{2} e^{4 k \sigma}=-\frac{4(2 k-1)^{2}}{2 k-3} X_{1}(\sigma)^{2} .
$$

This is impossible if $k \geq 2$, since $X_{1}(\sigma) \neq 0, \mu \neq 0$. The proof of the theorem is complete.

Remark 2.11. The same proof as above applies for the case $\operatorname{dim} M=4$ up to (2.14). However, from (2.14) and $i_{X_{1}} \Omega=0$, we now have

$$
\mathrm{d}^{\mathcal{H}}\left(\lambda^{-2}\right)=* \mathcal{H} \Omega
$$

where $\mathrm{d}^{\mathcal{H}}$ is the differential composed with the horizontal projection and $*_{\mathcal{H}}$ is the Hodge star-operator on $\left(\mathcal{H},\left.h\right|_{\mathcal{H}}\right)$ with respect to some orientation of $\mathcal{H}$.

From the proof of Theorem 2.8 it follows that if $\left(M^{4}, g\right)$ is a 4-dimensional Einstein manifold and $\varphi:\left(M^{4}, g\right) \rightarrow\left(N^{3}, h\right)$ is a submersive harmonic morphism to a Riemannian 3 -manifold which is not of type 1 or of type 2 (i.e. $\mathcal{V}=\operatorname{ker} \varphi_{*}$ is neither Riemannian, nor geodesic with integrable horizontal distribution), then the "monopole equation" (2.15) must hold and we obtain a third type of harmonic morphism [21] (see [20, Theorem 3.4.4]); note that the proof in 21] is different.

\section{HARMONIC MORPHISMS WITH ONE-DIMENSIONAL FIBRES BETWEEN EINSTEIN MANIFOLDS}

In this section, we refine Theorem 2.8 in the case where the codomain is also Einstein.

Theorem 3.1. Let $\varphi:\left(M^{n+1}, g\right) \rightarrow\left(N^{n}, h\right)$ be a surjective harmonic morphism with connected fibres between Einstein manifolds of dimension $n+1$ and $n(n \geq 4)$. Then, up to homotheties, one of the following assertions holds:

(i) $\varphi$ is a Riemannian submersion with geodesic fibres onto an almost Kähler manifold and $\Omega$ is the pull-back of its Kähler form (in particular, $\operatorname{dim} M$ is odd);

(ii) $\varphi$ is a horizontally homothetic submersion with geodesic fibres orthogonal to an umbilical foliation by hypersurfaces.

Remark 3.2. Note that any $x \in M$ has an open neighbourhood $U$ such that $\left.\varphi\right|_{U}$ has connected fibres. Furthermore, if $M$ is compact (more generally, if $\mathcal{H}$ is an Ehresmann connection) any harmonic morphism $\varphi:\left(M^{n+1}, g\right) \rightarrow\left(N^{n}, h\right)(n \geq 4)$ can be factorised into a harmonic morphism with connected fibres followed by a Riemannian covering [23].

Proof of Theorem 3.1. Let $n=\operatorname{dim} N$. If $\Omega=0$, then from (2.2) it follows that $X(\sigma) Y(\sigma)=0$ for any pair of orthogonal vectors $X$ and $Y$ where $\lambda=e^{\sigma}$ is the dilation of $\varphi$. Hence $X(\sigma)=0$ for any horizontal vector $X$, which implies that (ii) holds.

From now on we shall assume that $\Omega$ is nowhere zero. In particular, (ii) does not hold and hence, by Theorem [2.8, the fibres of $\varphi$ form a Riemannian foliation locally generated by Killing vector fields. Therefore $\lambda=e^{\sigma}$ is basic and thus both $\lambda$ and $\sigma$ are pull-backs by $\varphi$ of functions on $N$; we shall denote these two functions by the same letters $\lambda$ and $\sigma$. 
We have ${ }^{M}$ Ricci $=c^{M} g,{ }^{N}$ Ricci $=c^{N} h$ and we shall denote by the same letter $h$ both the metric on $N$ and the metric on $M$ with respect to which $\varphi$ is a Riemannian submersion with geodesic fibres.

To complete the proof we must show that (i) holds on an open subset of $M$.

From (2.2) we get

$$
\frac{1}{2} e^{(2 n-2) \sigma} h\left(i_{X} \Omega, i_{Y} \Omega\right)=-(n-1)(n-2) X(\sigma) Y(\sigma)
$$

for any pair $X, Y$ of orthogonal horizontal vectors.

As in the proof of Theorem 2.8, there is an open subset $U$ of $M$ on which we can consistently diagonalise $\Omega^{2}$ with respect to a basic orthonormal frame $\left\{X_{1}, \ldots, X_{n}\right\}$ of $(\mathcal{H}, h)$. Then, from (3.1) it follows that $X_{i}(\sigma) X_{j}(\sigma)=0$ for any $i \neq j$. It follows that either $X_{i}(\sigma)=0$ for all $i=1, \ldots, n$ or, after renumbering if necessary, on some open subset we have $X_{2}(\sigma)=\ldots=X_{n}(\sigma)=0$ and $X_{1}(\sigma)$ is not identically zero.

From (2.7) we obtain $\mu_{i}=\mu_{j}$ for any $i, j=2, \ldots, n$. Hence $\mu_{i}=\mu(i=2, \ldots, n)$ for some nonnegative function $\mu$ with $\mu^{2}$ smooth. From (2.7) we also get

$$
-\frac{1}{2} e^{(2 n-2) \sigma}\left(\mu_{1}^{2}-\mu_{2}^{2}\right)=(n-1)(n-2) X_{1}(\sigma)^{2} .
$$

Suppose that $X_{1}(\sigma)=0$ (equivalently, $\varphi$ has geodesic fibres), then $\sigma$ is constant and $\mu_{i}=\mu$ for $i=1, \ldots, n$. Moreover, from (2.2) it follows that $\mu=$ constant. Hence $n$ must be even and $\Omega$ is $\mu$ times the Kähler form of an almost Hermitian structure on $(N, h)$. Since $\mathrm{d} \Omega=0$, this structure is almost Kähler giving assertion (i).

Suppose instead that $X_{1}(\sigma) \neq 0$. We shall obtain a contradiction. We have $\mu_{1} \neq \mu_{2}$ and hence, because $\Omega \neq 0$ is skew-symmetric, $\mu_{1}=0$ and $\mu \neq 0$. Thus, $h\left(i_{X_{1}} \Omega, i_{X_{1}} \Omega\right)=\mu_{1}^{2}=0$ which implies $i_{X_{1}} \Omega=0$. Together with $X_{i}(\sigma)=0$ $(i=2, \ldots, n)$, this gives $i_{\operatorname{grad} \sigma} \Omega=0$. Also, (3.2) becomes

$$
\frac{1}{2} e^{(2 n-2) \sigma} \mu^{2}=(n-1)(n-2) X_{1}(\sigma)^{2},
$$

which can be written as

$$
(n-1) \mu^{2}=2(n-2) X_{1}\left(\lambda^{1-n}\right)^{2} .
$$

Since $\varphi$ is a harmonic morphism, by the chain rule (see, for example, [5, Section $3.2]$ ), we have that

$$
\Delta^{M}(f \circ \varphi)=\lambda^{2} \Delta^{N} f \circ \varphi
$$

for any smooth function $f$ on $N$.

From (2.6) we get that

$$
c^{M} e^{-2 \sigma}=c^{N}-\Delta^{N} \sigma-(n-1)(n-2) X_{1}(\sigma)^{2} .
$$

From (2.4) we get that

$$
c^{M} e^{-2 \sigma}=(n-2) \Delta^{N} \sigma+\frac{1}{4} e^{(2 n-2) \sigma}|\Omega|_{h}^{2} .
$$

Because $\mu \neq 0$, as before, we have that $n$ must be odd. Then we can choose the frame $\left\{X_{1}, \ldots, X_{n}\right\}$ such that, with respect to this frame, $\Omega$ is given by (2.9). In particular, $|\Omega|_{h}^{2}=(n-1) \mu^{2}$ and (3.6) becomes

$$
c^{M} e^{-2 \sigma}=(n-2) \Delta^{N} \sigma+\frac{1}{4}(n-1) e^{(2 n-2) \sigma} \mu^{2} .
$$

The relations (3.3), (3.5), (3.7) give

$$
\frac{1}{2}(n-1)(n-2)(n-3) X_{1}(\sigma)^{2}=-(n-1) e^{-2 \sigma} c^{M}+(n-2) c^{N} .
$$


Because $n \geq 4$, relations (3.3), (3.8) and the fact that $X_{i}(\sigma)=0 \quad(i=2, \ldots, n)$ imply $X_{i}(\mu)=0 \quad(i=2, \ldots, n)$.

Note that (3.8) can be written as follows:

$$
\frac{n-3}{2} X_{1}(\lambda)^{2}=-\frac{c^{M}}{n-2}+\frac{c^{N}}{n-1} \lambda^{2} .
$$

From now on $\Omega, \mu$ and $X_{j}, j=1, \ldots, n$, will be viewed as objects on $N$. From (2.3) and $i_{\text {grad } \sigma} \Omega=0$ we get that ${ }^{h} \mathrm{~d}^{*} \Omega=0$.

Let $\mathcal{F}$ be the foliation formed on $N$ by the level hypersurfaces of $\sigma$. Note that $X_{2}, \ldots, X_{n}$ are tangent to $\mathcal{F}$ and $X_{1}$ is normal to $\mathcal{F}$. Also, $\mu$ is constant along the leaves of $\mathcal{F}$ and, from (2.9), it follows that $\Omega$ restricted to any leaf $L$ of $\mathcal{F}$ is $\mu$ times the Kähler form of an almost Hermitian structure $J$ on $\left(L,\left.h\right|_{L}\right)$. But $\mathrm{d} \Omega=0$ and the constancy of $\mu$ along $L$ imply that $J$ is actually an almost Kähler structure on $\left(L,\left.h\right|_{L}\right)$. In particular, $\operatorname{div} \mathcal{F}\left(\left.\mu^{-1} \Omega\right|_{L}\right)=0$ which is equivalent to $\operatorname{div}_{\mathcal{F}}\left(\left.\Omega\right|_{L}\right)=0$ where $\operatorname{div}_{\mathcal{F}} \Omega=\sum_{j=2}^{n}\left((\mathcal{F} \nabla)_{X_{j}} \Omega\right)\left(X_{j}, \cdot\right)$ (as before, we denote by the same letters the distributions $\mathcal{F}$ and $\mathcal{F}^{\perp}$ and the orthogonal projections onto them). After a short calculation (similar to the one used in the proof of Theorem 2.8 to find $\left.\left({ }^{h} \mathrm{~d}^{*} \Omega\right)\left(X_{1}\right)\right)$, by using the fact that $\mathcal{F}$ is integrable, we get that $\left(\operatorname{div}_{\mathcal{F}} \Omega\right)\left(X_{1}\right)=0$. Hence $\operatorname{div}_{\mathcal{F}} \Omega=0$.

We claim that $\operatorname{div} \mathcal{F} \Omega=0$ and $\operatorname{div} \Omega=-{ }^{h} \mathrm{~d}^{*} \Omega=0$ imply that $\mathcal{F}^{\perp}$ has geodesic fibres. Indeed, we shall see that

$$
\operatorname{div} \Omega=\operatorname{div}_{\mathcal{F}} \Omega-\mu \operatorname{trace}\left(B^{\perp}\right)^{b} \circ J
$$

where $B^{\perp}$ is the second fundamental form of $\mathcal{F}^{\perp}$. Hence $\operatorname{trace}\left(B^{\perp}\right)=0$, i.e., $\mathcal{F}^{\perp}$ is geodesic.

Next, we prove (3.10). We have

$$
\begin{aligned}
\operatorname{div} \Omega & =\sum_{j=1}^{n}\left(\nabla_{X_{j}} \Omega\right)\left(X_{j}, \cdot\right) \\
& =\left(\nabla_{X_{1}} \Omega\right)\left(X_{1}, \cdot\right)+\sum_{j=2}^{n}\left(\nabla_{X_{j}} \Omega\right)\left(X_{j}, \cdot\right) \\
& =\left(\nabla_{X_{1}} \Omega\right)\left(X_{1}, \cdot\right)+\sum_{j=2}^{n}\left((\mathcal{F} \nabla)_{X_{j}} \Omega\right)\left(X_{j}, \cdot\right)+\sum_{j=2}^{n}\left(\left(\mathcal{F}^{\perp} \nabla\right)_{X_{j}} \Omega\right)\left(X_{j}, \cdot\right) \\
& =\left(\nabla_{X_{1}} \Omega\right)\left(X_{1}, \cdot\right)+\operatorname{div} \mathcal{F} \Omega+\sum_{j=2}^{n}\left(\left(\mathcal{F}^{\perp} \nabla\right)_{X_{j}} \Omega\right)\left(X_{j}, \cdot\right) .
\end{aligned}
$$

Obviously, $\left(\nabla_{X_{1}} \Omega\right)\left(X_{1}, X_{1}\right)=0$, and $i_{X_{1}} \Omega=0$ implies that $\left(\left(\mathcal{F}^{\perp} \nabla\right)_{X_{j}} \Omega\right)\left(X_{j}, X_{1}\right)=$ 0 for any $j=2, \ldots, n$ (recall that $\left\{X_{1}\right\}$ is a local frame for $\mathcal{F}^{\perp}$ ). Also $i_{X_{1}} \Omega=0$ and $X_{j}(\mu)=0(j=2, \ldots, n)$ imply that $\left(\left(\mathcal{F}^{\perp} \nabla\right)_{X_{j}} \Omega\right)\left(X_{j}, X_{k}\right)=0$ for any $j, k=2, \ldots, n$.

For $j=1, \ldots,(n-1) / 2$, we have

$$
\begin{aligned}
\left(\nabla_{X_{1}} \Omega\right)\left(X_{1}, X_{2 j}\right) & =-\Omega\left(\nabla_{X_{1}} X_{1}, X_{2 j}\right) \\
& =-h\left(\nabla_{X_{1}} X_{1}, X_{2 j+1}\right) \Omega\left(X_{2 j+1}, X_{2 j}\right) \\
& =-\mu h\left(\operatorname{trace}\left(B^{\perp}\right), J X_{2 j}\right) .
\end{aligned}
$$


Similarly,

$$
\left(\nabla_{X_{1}} \Omega\right)\left(X_{1}, X_{2 j+1}\right)=-\mu h\left(\operatorname{trace}\left(B^{\perp}\right), J X_{2 j+1}\right) .
$$

Relation (3.10) now follows from (3.11), 3.12) and (3.13).

From $i_{X_{1}} \Omega=0$ and $\mathrm{d} \Omega=0$ it follows that $\mathcal{L}_{X_{1}} \Omega=\mathrm{d} i_{X_{1}} \Omega+i_{X_{1}} \mathrm{~d} \Omega=0$. Hence $\Omega$ is basic for $\mathcal{F}^{\perp}$. It follows also that $J$ is basic for $\mathcal{F}^{\perp}$, equivalently, $\mathcal{F}^{\perp}$ is defined by horizontally holomorphic submersions (see the Appendix for the definition of horizontally holomorphic submersion). Moreover, by taking on the codomain of such a submersion the metric induced by any leaf of $\mathcal{F}$, from Proposition A.1 of the Appendix we get that $\mathcal{F}^{\perp}$ is defined by horizontally holomorphic harmonic submersions.

It is easy to see that $\Omega(J X, Y)=\mu h(X, Y)$ for any $X, Y \in \mathcal{F}$. Because both $\Omega$ and $J$ are basic for $\mathcal{F}^{\perp}$, we get that $\mathcal{F}^{\perp}$ is a conformal foliation with dilation $\mu^{1 / 2}$. Moreover, because $\mu$ is constant along the leaves of $\mathcal{F}$, we get that $\mathcal{F}^{\perp}$ is a homothetic foliation. Thus, $\mathcal{F}^{\perp}$ is locally defined by horizontally homothetic submersions $\psi:(N, h) \rightarrow(P, k)$ with geodesic fibres (equivalently, $\mathcal{F}^{\perp}$ corresponds, locally, to a warped product decomposition of $(N, h))$. Moreover, $(P, k)$ is an Einstein $(n-2)$-manifold (apply, for example, [6, (9.109)]). From [6, (9.109)] it follows that $\mu$ satisfies the following equation:

$$
X_{1}\left(\mu^{-1 / 2}\right)=\frac{c^{P}}{n-2}-\frac{c^{N}}{n-1} \mu^{-1}
$$

where the Ricci tensor of $(P, k)$ is given by ${ }^{P}$ Ricci $=c^{P} k$.

After a straightforward elementary calculation we get that the equations (3.4), (3.9) and (3.14) are incompatible and the proof follows.

Remark 3.3. 1) Note that, if $\operatorname{dim} N=3$, then from the above proof we get that $i_{\operatorname{grad} \lambda} \Omega=0$ and (3.4); equivalently, $\lambda^{-2}$ and $\Omega$ are related by the $S^{1}$-monopole equation (see 20], 21] for this case).

2) Both case (i) and case (ii) of Theorem 3.1 are related to well-known constructions of Einstein metrics (see [6, Chapter 9]).

\section{APPENDIX A. A REMARK ON HORIZONTALlY HOLOMORPHIC SUBMERSIONS}

Let $\mathcal{V}$ be a foliation on the Riemannian manifold $(M, g)$. Assume that on the horizontal distribution $\mathcal{H}=\mathcal{V}^{\perp}$ there exists an almost Hermitian structure $J$ which is a basic tensor field for $\mathcal{V}$. Then, $\mathcal{V}$ could be locally defined by submersions $\varphi:\left(U,\left.g\right|_{U}\right) \rightarrow(N, \breve{J})$ onto almost complex manifolds such that, at each point $x \in U$, the differential $\left.\varphi_{*, x}\right|_{\mathcal{H}_{x}}:\left(\mathcal{H}_{x}, J_{x}\right) \rightarrow\left(T_{\varphi(x)} N, \breve{J}_{\varphi(x)}\right)$ is complex linear. We shall call such submersions horizontally holomorphic (see [17] for alternative terminology). Obviously, any holomorphic submersion from an almost Hermitian manifold to an almost complex manifold is horizontally holomorphic, but there exist simple examples of horizontally holomorphic submersions (e.g., orthogonal projections, the Hopf fibrations) which are not holomorphic maps.

Let $\left(M^{m}, g\right)$ be a Riemannian manifold endowed with a pair of complementary orthogonal distributions $\mathcal{H}$ and $\mathcal{V}$ of dimension $n$ and $m-n$, respectively. For a $(p, q)$-tensor field $T$ on $M$ the horizontal divergence $\operatorname{div}_{\mathcal{H}} T$ is the $(p, q-1)$-tensor field given by

$$
\operatorname{div}_{\mathcal{H}} T=\sum_{a=1}^{n} i_{X_{a}}\left((\mathcal{H} \nabla)_{X_{a}} T\right)
$$


where $\left\{X_{a}\right\}_{a=1, \ldots, n}$ is a local orthonormal frame for $\mathcal{H}$.

Proposition A.1. Let $\varphi:(M, g) \rightarrow(N, h, J)$ be a horizontally holomorphic submersion to an almost Hermitian manifold. Then

$$
J\left(\operatorname{trace}\left(B^{\mathcal{V}}\right)\right)+J(\tau(\varphi))+\operatorname{trace}_{g} \varphi^{*}(\stackrel{N}{\nabla} J)-\operatorname{div}_{\mathcal{H}} J=0
$$

where $\mathcal{V}=\operatorname{ker} \varphi_{*}, B^{\mathcal{V}}$ is the second fundamental form of $\mathcal{V}, \stackrel{N}{\nabla}$ is the Levi-Civita connection of $(N, h)$ and $\tau(\varphi)$ is the tension field of $\varphi$.

Proof. Clearly by identifying $\varphi^{*}(T N)$ with $\mathcal{H}$ the $\varphi^{*}(T N)$-valued one-form induced by $\varphi_{*}$ becomes the "horizontal" projection $\mathcal{H}$.

Let $X, Y$ be horizontal vector fields. With $\nabla$ the connection on $\varphi^{*}(T N) \otimes T^{*} M$ we can write

$$
\begin{aligned}
\left(\nabla \varphi_{*}\right)(X, J Y) & =\varphi^{*}(\stackrel{N}{\nabla})_{X}(\mathcal{H} J Y)-\mathcal{H}\left(\stackrel{M}{\nabla}_{X}(J Y)\right) \\
& =\varphi^{*}(\stackrel{N}{\nabla})_{X}(J Y)-(\mathcal{H} \stackrel{M}{\nabla})_{X}(J Y) \\
& =\left(\varphi^{*}(\stackrel{N}{\nabla})_{X} J\right)(Y)+J\left(\varphi^{*}(\stackrel{N}{\nabla})_{X} Y\right)-\left(\left(\mathcal{H} \nabla^{M}\right)_{X} J\right)(Y)-J\left((\mathcal{H} \stackrel{M}{\nabla})_{X} Y\right) .
\end{aligned}
$$

Hence

$$
\left(\nabla \varphi_{*}\right)(X, J Y)=J\left(\left(\nabla \varphi_{*}\right)(X, Y)\right)+\varphi^{*}(\stackrel{N}{\nabla} J)_{X} Y-((\mathcal{H} \stackrel{M}{\nabla}) J)_{X} Y .
$$

Let $\left\{X_{a}\right\}$ be a local orthonormal frame for $\left(\mathcal{H}, g_{\mathcal{H}}\right)$. From (A.1) we get

$$
J\left(\sum_{a}\left(\nabla \varphi_{*}\right)\left(X_{a}, X_{a}\right)\right)+\operatorname{trace}_{g} \varphi^{*}(\stackrel{N}{\nabla} J)-\operatorname{div}_{\mathcal{H}} J=0 .
$$

As is well-known (and easy to prove) $\left(\nabla \varphi_{*}\right)(V, W)=-B^{\mathcal{V}}(V, W)$ for any vertical vectors $V, W$. The proof follows from this fact and (A.2).

Remark A.2. 1) From Proposition A.1 we could obtain a result similar to the one of 3 ] which relates the condition for a horizontally conformal submersion to be harmonic (and hence, a harmonic morphism) and the property that its fibres be minimal ([17]).

2) A proof similar to the one above could be obtained for the following formula of A. Lichnerowicz [16] for a holomorphic map $\varphi:(M, g, J) \rightarrow(N, h, J)$ between almost Hermitian manifolds:

$$
J(\tau(\varphi))+\operatorname{trace}_{g} \varphi^{*}(\stackrel{N}{\nabla} J)-\operatorname{div} J=0 .
$$

It follows easily from (A.3) that any holomorphic map from a cosymplectic manifold to a $(1,2)$-symplectic manifold is harmonic [16] (cf. [13]).

\section{REFERENCES}

[1] P. Baird, Harmonic maps with symmetry, harmonic morphisms and deformations of metrics, Research Notes in Mathematics, 87, Pitman (Advanced Publishing Program), Boston, Mass.London, 1983. MR 85i:58038

[2] P. Baird, Harmonic morphisms and circle actions on 3- and 4-manifolds, Ann. Inst. Fourier (Grenoble), 40 (1990) 177-212. MR 91e:57025]

[3] P. Baird, J. Eells, A conservation law for harmonic maps, Geometry Symposium. Utrecht 1980, Lecture Notes in Math. 894, Springer-Verlag, Berlin, Heidelberg, New York, 1981, 1-25. MR 83i:58031 
[4] P. Baird, J.C. Wood, Harmonic morphisms, Seifert fibre spaces and conformal foliations, Proc. London Math. Soc., 64 (1992) 170-196. MR 93c:58051]

[5] P. Baird, J.C. Wood, Harmonic morphisms between Riemannian manifolds, London Math. Soc. Monogr. (N.S.), Oxford Univ. Press (to appear).

[6] A.L. Besse, Einstein manifolds, Ergebnisse der Mathematik und ihrer Grenzgebiete (3), 10, Springer-Verlag, Berlin-New York, 1987. MR 88f:53087

[7] R.L. Bryant, Harmonic morphisms with fibers of dimension one, Comm. Anal. Geom., 8 (2000) 219-265. MR 2001i:53101

[8] J. Eells, L. Lemaire, Selected topics in harmonic maps, CBMS Regional Conference Series in Mathematics, 50, Published for the Conference Board of the Mathematical Sciences, Washington, DC; by the American Mathematical Society, Providence, RI, 1983. MR 85g:58030

[9] B. Fuglede, Harmonic morphisms between Riemannian manifolds, Ann. Inst. Fourier (Grenoble), 28 (1978) 107-144. MR 80h:58023

[10] M. Golubitsky, V. Guillemin, Stable mappings and their singularities, Graduate Texts in Mathematics, Vol. 14, Springer-Verlag, New York-Heidelberg, 1973. MR 49:6269

[11] S. Gudmundsson, The Geometry of Harmonic Morphisms, Ph.D. Thesis, University of Leeds, 1992.

[12] S. Gudmundsson, The Bibliography of Harmonic Morphisms, http://www.maths.1th.se/ matematiklu/personal/sigma/harmonic/bibliography.html

[13] S. Gudmundsson, J.C. Wood, Harmonic morphisms between almost Hermitian manifolds, Boll. Un. Mat. Ital. B (7), 11 (1997) 185-197. MR 98i:58069

[14] T. Ishihara, A mapping of Riemannian manifolds which preserves harmonic functions, J. Math. Kyoto Univ., 19 (1979) 215-229. MR 80k:58045

[15] S. Kobayashi, K. Nomizu, Foundations of differential geometry, I, II, Interscience Tracts in Pure and Applied Math. 15, Interscience Publ., New York, London, Sydney, 1963, 1969. MR 27:2945: MR 38:6501

[16] A. Lichnerowicz, Applications harmoniques et variétés kähleriennes, Symposia Mathematica, Vol. III (INDAM, Rome, 1968/1969), 341-402. MR 41:7598

[17] E. Loubeau, X. Mo, Pseudo horizontally weakly conformal maps from Riemannian manifolds into Kähler manifolds, preprint, University of Brest, 2000.

[18] X. Mo, Horizontally conformal maps and harmonic morphisms, Chinese Journal of Contemporary Mathematics, 17 (1996) 245-252. MR 97m:58055

[19] R. Pantilie, Harmonic morphisms with one-dimensional fibres, Internat. J. Math., 10 (1999) 457-501. MR 2000d:53100

[20] R. Pantilie, Submersive harmonic maps and morphisms, Ph.D. Thesis, University of Leeds, 2000.

[21] R. Pantilie, Harmonic morphisms with 1-dimensional fibres on 4-dimensional Einstein manifolds, Comm. Anal. Geom., (to appear).

[22] R. Pantilie, J.C. Wood, New results on harmonic morphisms with one-dimensional fibres, Bull. Math. Soc. Sci. Math. Roumanie, 43 (2000), Volume in the memory of G. Vrânceanu, 355-365. MR 2002b:53107

[23] R. Pantilie, J.C. Wood, Topological restrictions for circle actions and harmonic morphisms, preprint, University of Leeds, 2000.

[24] R. Pantilie, J.C. Wood, A new construction of Einstein self-dual metrics, Asian J. Math. (to appear).

[25] B.L. Reinhart, Differential geometry of foliations. The fundamental integrability problem, Ergebnisse der Mathematik und Ihrer Grenzgebiete, 99, Springer-Verlag, Berlin-New York, 1983. MR 85i:53038

[26] N.E. Steenrod, The topology of fibre bundles, Princeton Mathematical Series 14, Princeton University Press, 1951. MR 12:522b

[27] J.C. Wood, Harmonic morphisms, foliations and Gauss maps, Complex differential geometry and non-linear differential equations, Contemp. Math. 49, Amer Math. Soc., Providence, RI, 1986, 145-183. MR 87i:58045

School of Mathematics, University of Leeds, Leeds LS2 9JT, England

E-mail address: r.pantilie@leeds.ac.uk

School of Mathematics, University of Leeds, Leeds LS2 9JT, England

E-mail address: j.c.wood@leeds.ac.uk . 